\title{
The emerging role of oestrogen-related receptor $\gamma$ as a regulator of energy metabolism
}

\author{
Varman T. Samuel
}

Received: 28 August 2014 / Accepted: 3 September 2014 / Published online: 26 September 2014

(C) Springer-Verlag (outside the USA) 2014

\begin{abstract}
Coordinating energy supply with use is critical for survival and is tightly regulated. There is growing evidence that the oestrogen-related receptors (ERRs), a family of orphan nuclear receptors, play key roles in this regulation, ERR $\gamma$ being the most recently discovered member of this family. Hepatic expression and activity of ERR $\gamma$ is induced by fasting and repressed in response to feeding. Work reported by Kim and colleagues (DOI: 10.1007/s00125-014-3366-x) dissects the mechanism for the latter observation. Taken together with prior studies by this group and others, there is emerging evidence that this protein helps coordinate metabolism and may be a potential therapeutic target.
\end{abstract}

Keywords Akt/PKB $\cdot$ Hepatic gluconeogenesis $\cdot$ Insulin receptor signalling $\cdot$ Nuclear hormone receptor .

Phosphorylation

Abbreviations
$\begin{array}{ll}\text { CREB } & \text { cAMP response element-binding protein } \\ \text { ERR } & \text { Oestrogen-related receptor } \\ \text { FOXO1 } & \text { Forkhead box protein O1 } \\ \text { PKB } & \text { Protein kinase B } \\ \text { PCK1 } & \text { Phosphoenolpyruvate carboxykinase, cytosolic }\end{array}$

Animals continually counter energy needs against energy availability and have evolved systems for storing energy when food is abundant and later utilising that energy to match metabolic demands. Gluconeogenesis is one of the tools in

V. T. Samuel $(\bowtie)$

Department of Internal Medicine, Yale University School of

Medicine, New Haven, CT 06520, USA

e-mail: Varman.samuel@yale.edu

V. T. Samuel

Veterans Affairs Medical Center, West Haven, CT, USA this continual balancing act. As the name implies, gluconeogenesis produces glucose from 3- and 4-carbon precursors and helps ensure a steady supply of glucose. Most critically, gluconeogenesis provides glucose for the brain, where neurons and glia preferentially utilise glucose for energy.

The regulation of gluconeogenesis is necessarily robust and plastic. This is exemplified by the regulation of gluconeogenesis under the diametric conditions of feeding and fasting. Normally, endogenous glucose production is completely suppressed immediately following a meal [1]. However, with fasting, gluconeogenesis enables the body to maintain endogenous gluconeogenesis production, accounting for nearly all glucose production as glycogen is depleted during prolonged fasting [2]. The flux of carbons through this pathway is regulated at many levels. Though much attention is given to the transcriptional control of enzyme expression in response to hormonal and neural inputs, these enzymes are also subject to allosteric control and post-translational modifications. And, of course, substrate availability also influences the rate of gluconeogenesis.

However, the enzymes of this pathway also participate in other key metabolic roles. The gluconeogenic pathway is important for hepatic glycogen synthesis. Termed the 'indirect pathway', trioses produced by glycolysis are taken up by the liver, resynthesised into glucose 6-phosphate and stored as glycogen. The indirect pathway accounts for approximately $30-50 \%$ of hepatic glycogen synthesised after an oral glucose load [3]. In addition, some of the gluconeogenic enzymes are involved in glyceroneogenesis [4]. The synthesis of glycerol 3-phosphate, the 3-carbon backbone on which fatty acids are sequentially esterified to form triacylglycerol is most apparent in white adipose tissue, especially when dietary lipids are in excess [5]. Finally, some gluconeogenic enzymes have been implicated in regulating mitochondrial 
energy metabolism [6, 7]. Thus, considered more broadly, the enzymes involved in gluconeogenesis help to regulate carbon flux to match the changing metabolic needs of the cell and entire organism.

Many studies have focused on the transcriptional regulation of gluconeogenesis, identifying an intricate web of signals that connect hormone signalling to the transcription of the genes encoding key enzymes, such as phosphoenolpyruvate carboxykinase, cytosolic (PKC1) and glucose-6-phosphatase. In a very simplistic model to illustrate this concept, insulin and glucagon act in opposition to suppress and activate transcription of PCK1. Insulin signalling leads to the activation of protein kinase $\mathrm{B}$ (PKB or Akt), which then phosphorylates forkhead box protein O1 (FOXO1), leading to nuclear exclusion and degradation and resulting in decreased transcription of FOXO1 targets, such as PCK1 [8]. In contrast, glucagon promotes phosphorylation of cAMP response elementbinding protein (CREB), enhancing its ability to increase expression of key target genes, including $P C K 1$ [9]. The promoter for $P C K 1$ is complex, with multiple elements that integrate multiple hormone, neural and nutrient signals via the binding of a diverse array of transcription factors and co-factors (e.g. glucocorticoid receptor, thyroid hormone receptor, sterol regulatory element binding protein 1 [SREBP1], signal transducer and activator of transcription 3 [STAT3], etc.) [10].

Oestrogen-related receptors (ERRs), a family of three orphan nuclear receptors, are among the proteins that regulate the expression of key metabolic enzymes, including gluconeogenic enzymes. ERR $\alpha$ suppresses expression of $P C K 1$ by interfering with PGC- $1 \alpha$ binding and decreasing $P C K 1$ transcription [11]. In contrast, ERR $\gamma$ has been implicated in increasing PCK1 expression. Though ERRs may be regulated by as yet unknown endogenous ligands, ERR $\gamma$ is also subject to regulation at transcriptional and posttranslational (i.e. phosphorylation) levels. In a previous report, Kim and colleagues demonstrate that ERR $\gamma$ expression is induced by glucagon in a CREB regulated transcription coactivator 2 (CRTC2)-CREB-dependent fashion [12]. This accounts for the observed increase in ERR $\gamma$ expression with fasting. ERR $\gamma$ then enhances gluconeogenesis directly by binding to ERR elements in the $P C K 1$ promoter. ERR $\gamma$ activity on the PCK1 promoter can be further enhanced by peroxisome proliferator-activated receptor $\gamma$ coactivator $1 \alpha$ $(\mathrm{PGC} 1 \alpha)$. Thus, ERR $\gamma$, along with other transcription factors, may serve to amplify the intracellular signal from glucagon to further increase expression of $P C K 1$ with fasting.

In the study published in this issue of Diabetologia, Kim and colleagues tackle the other side of ERR $\gamma$ regulation: how insulin may suppress ERR $\gamma$ activity via phosphorylation [13]. The studies are methodical and comprehensive. They demonstrate a requirement of insulin signalling to promote phosphorylation of S179 of ERR $\gamma$; mice lacking the insulin receptor or PKB fail to phosphorylate ERR $\gamma$. Similarly, in an insulin-resistant model ( $d b / d b$ mice), insulin injection fails to increase PKB and ERR $\gamma$ phosphorylation. This model accounts for the changes in ERR $\gamma$ phosphorylation seen with feeding.

Phosphorylation of ERR $\gamma$ at S179 occurs within the DNAbinding domain of this protein. Thus, phosphorylation reduces DNA binding, leading to a reduction in the nuclear content of ERR $\gamma$, with redistribution into the cytoplasm by a 14-3-3 chaperone. Kim and colleagues rigorously test this model in vitro using two mutant forms of ERR $\gamma$ : S179A, which cannot be phosphorylated, and S179D, which mimics the phosphorylated form. S179A has enhanced nuclear localisation and transcriptional activity and sustains transcription of gluconeogenic enzymes even in the presence of insulin. In comparison, S179D is relegated to the cytosol and has a markedly decreased ability to activate transcription from the Pck1 promoter.

This proposed mechanism was then tested in vivo. Transfecting mice with adenoviral vectors to increase the expression of ERR $\gamma$ S179A (but not wild-type ERR $\gamma$ ) increases the basal glucose concentration and the expression of both Pckl and Pdk4 (another target of ERR $\gamma$ ). Moreover, the authors observed that mice treated with Ad-ERR $\gamma$ S179A had an attenuated plasma glucose response to insulin, indicating some degree of insulin resistance. In addition, treatment of cells with GSK5182, an inverse agonist of ERR $\gamma$, decreased the ability of ERR $\gamma$ to activate the Pckl expression. Interestingly, this did not alter phosphorylation of ERR $\gamma$. These inhibitor studies are consistent with previous studies from this group in which they demonstrate that GSK5182 decreases basal endogenous glucose production (EGP) [14]. Thus, this work comprehensively examines the mechanisms whereby insulin action may act through ERR $\gamma$ to regulate $P c k l$ expression.

But, the emphasis on the regulation of Pckl does not fully capture the complexity of this system. First, though the regulation of Pckl expression is equated to the regulation of gluconeogenesis by many, this overstates the impact of PCK1 on gluconeogenesis. Liver-specific PCK1 deletion does not lead to hypoglycaemia [15]. Modest overexpression (twofold increase) does not lead to fasting hyperglycaemia [16]. Using a variety of PCK1 deletion models, Burgess and colleagues reported that this enzyme has relatively weak control over the rate of gluconeogenesis [17]. We have previously reported that although fasting hyperglycaemia is often attributed to increased PCK1, this is not actually evident in rodents or humans with type 2 diabetes mellitus [18]. Thus, altered regulation of PCK1 cannot solely account for fasting hyperglycaemia. Second, ERR $\gamma$ clearly has other roles in regulating insulin action. Kim and colleagues earlier demonstrated that ERR $\gamma$ activates expression of LIPIN1, a phosphatase that increases intracellular diacylglycerol content, which 
activates $\mathrm{PKC} \varepsilon$, leading to impaired insulin signalling [19]. Treatment of mice with GSK5182 decreases LIPIN1 expression, diacylglycerol content and activation of PKCE. In mice, this is associated with a reduction in basal glucose production, with improvements in both hepatic and peripheral insulin action. In the present study, the insulin resistance seen with overexpression of the S179A mutant could also be due to altered intracellular lipid metabolism and impairment of insulin signalling.

Thus, ERR $\gamma$ is another layer of a complex regulatory fabric evolved to balance hepatic glucose and lipid metabolism under myriad physiological conditions. In future studies, it will be important to assess how ERR $\gamma$ may impact hepatic glycogen metabolism (e.g. does it alter glycogen synthesis via the indirect pathway?) and lipid metabolism (e.g. can it alter glyceroneogenesis?). ERR $\gamma$ clearly regulates cellular metabolism in other tissues. For example, ERR $\gamma$-null mice die early in life as a result of cardiac defects and exhibit numerous alterations in the expression of genes involved in cardiac mitochondrial function [20]. ERR $\gamma$ expression demonstrates circadian rhythmicity in liver and brown adipose tissue but not white adipose tissue [21]. Future studies will hopefully provide a broader view of how ERR $\gamma$ may act to coordinate fuel supply and use across different organs. Finally, the elusive identity of the endogenous ligands for this family of proteins remains a challenge. The discovery of the endogenous ligands will enable a truer appreciation of the role these proteins play in regulating metabolism.

Though nature has equipped us with versatile systems to adapt energy availability to metabolic demands, energy intake now exceeds energy demand in many industrialised societies, with resulting epidemics of obesity and type 2 diabetes. Increases in gluconeogenesis account for fasting hyperglycaemia and contribute to postprandial hyperglycaemia $[22,23]$. To date, metformin is the only effective and widely approved oral therapy that decreases hepatic gluconeogenesis [24]. The changes following pharmacological inhibition by GSK5182 are compelling and suggest that ERR $\gamma$ may be a potential novel therapeutic target to decrease gluconeogenesis and potentially improve insulin sensitivity. The successful development of ERR $\gamma$ as a target will require more comprehensive in vivo metabolic studies, with careful assessments of cellular metabolism and insulin action in multiple tissues. Fortunately, these initial studies set a solid foundation upon which this development can proceed.

Acknowledgements VTS is supported by a VA Merit Award (I01 BX000901)

Duality of interest The author declares that there is no duality of interest associated with this manuscript.

Contribution statement VTS is the sole contributor to this commentary.

\section{References}

1. Firth RG, Bell PM, Marsh HM, Hansen I, Rizza RA (1986) Postprandial hyperglycemia in patients with noninsulin-dependent diabetes mellitus. Role of hepatic and extrahepatic tissues. J Clin Invest 77:1525-1532

2. Rothman DL, Magnusson I, Katz LD, Shulman RG, Shulman GI (1991) Quantitation of hepatic glycogenolysis and gluconeogenesis in fasting humans with ${ }^{13} \mathrm{C}$ NMR. Science (New York, NY) 254: 573-576

3. Taylor R, Magnusson I, Rothman DL et al (1996) Direct assessment of liver glycogen storage by ${ }^{13} \mathrm{C}$ nuclear magnetic resonance spectroscopy and regulation of glucose homeostasis after a mixed meal in normal subjects. J Clin Invest 97:126-132

4. Reshef L, Olswang Y, Cassuto H et al (2003) Glyceroneogenesis and the triglyceride/fatty acid cycle. J Biol Chem 278:30413-30416

5. Bederman IR, Foy S, Chandramouli V, Alexander JC, Previs SF (2009) Triglyceride synthesis in epididymal adipose tissue: contribution of glucose and non-glucose carbon sources. J Biol Chem 284: 6101-6108

6. Berglund ED, Kang L, Lee-Young RS et al (2010) Glucagon and lipid interactions in the regulation of hepatic AMPK signaling and expression of PPAR $\alpha$ and FGF21 transcripts in vivo. Am J Physiol Endocrinol Metab 299:E607-E614

7. Burgess SC, Hausler N, Merritt M et al (2004) Impaired tricarboxylic acid cycle activity in mouse livers lacking cytosolic phosphoenolpyruvate carboxykinase. J Biol Chem 279:48941-48949

8. Nakae J, Kitamura T, Silver DL, Accili D (2001) The forkhead transcription factor Foxol (Fkhr) confers insulin sensitivity onto glucose-6-phosphatase expression. J Clin Invest 108:1359-1367

9. Koo SH, Flechner L, Qi L et al (2005) The CREB coactivator TORC2 is a key regulator of fasting glucose metabolism. Nature 437:1109-1111

10. Chakravarty K, Cassuto H, Reshef L, Hanson RW (2005) Factors that control the tissue-specific transcription of the gene for phosphoenolpyruvate carboxykinase-C. Crit Rev Biochem Mol Biol 40:129-154

11. Herzog B, Cardenas J, Hall RK et al (2006) Estrogen-related receptor alpha is a repressor of phosphoenolpyruvate carboxykinase gene transcription. J Biol Chem 281:99-106

12. Kim D-K, Ryu D, Koh M et al (2012) Orphan nuclear receptor estrogen-related receptor $\gamma(\mathrm{ERR} \gamma)$ is key regulator of hepatic gluconeogenesis. J Biol Chem 287:21628-21639

13. Kim D-K, Kim Y-H, Hynx D et al (2014) PKB/Akt phosphorylation of ERR $\gamma$ contributes to insulin-mediated inhibition of hepatic gluconeogenesis. Diabetologia. doi:10.1007/s00125-014-3366-x

14. Kim D-K, Gang G-T, Ryu D et al (2013) Inverse agonist of nuclear receptor ERR $\gamma$ mediates antidiabetic effect through inhibition of hepatic gluconeogenesis. Diabetes 62:3093-3102

15. She P, Shiota M, Shelton KD, Chalkley R, Postic C, Magnuson MA (2000) Phosphoenolpyruvate carboxykinase is necessary for the integration of hepatic energy metabolism. Mol Cell Biol 20:6508-6517

16. Sun Y, Liu S, Ferguson S et al (2002) Phosphoenolpyruvate carboxykinase overexpression selectively attenuates insulin signaling and hepatic insulin sensitivity in transgenic mice. J Biol Chem 277:23301-23307

17. Burgess SC, He T, Yan Z et al (2007) Cytosolic phosphoenolpyruvate carboxykinase does not solely control the rate of hepatic gluconeogenesis in the intact mouse liver. Cell Metab $5: 313-320$

18. Samuel VT, Beddow SA, Iwasaki T et al (2009) Fasting hyperglycemia is not associated with increased expression of PEPCK or G6Pc in patients with type 2 diabetes. Proc Natl Acad Sci U S A 106:12121-12126 
19. Kim D-K, Kim JR, Koh M et al (2011) Estrogen-related receptor $\gamma$ $(\mathrm{ERR} \gamma)$ is a novel transcriptional regulator of phosphatidic acid phosphatase, LIPIN1, and inhibits hepatic insulin signaling. J Biol Chem 286:38035-38042

20. Alaynick W, Kondo RP, Xie W et al (2007) ERR $\gamma$ directs and maintains the transition to oxidative metabolism in the postnatal heart. Cell Metab 6:13-24

21. Yang X, Downes M, Yu RT et al (2006) Nuclear receptor expression links the circadian clock to metabolism. Cell 126:801-810
22. Magnusson I, Rothman DL, Katz LD, Shulman RG, Shulman GI (1992) Increased rate of gluconeogenesis in type II diabetes mellitus. A ${ }^{13} \mathrm{C}$ nuclear magnetic resonance study. J Clin Invest 90:1323-1327

23. Woerle HJ, Szoke E, Meyer C et al (2006) Mechanisms for abnormal postprandial glucose metabolism in type 2 diabetes. Am J Physiol 290:E67-E77

24. Hundal RS, Krssak M, Dufour S et al (2000) Mechanism by which metformin reduces glucose production in type 2 diabetes. Diabetes 49:2063-2069 